\title{
Blending learning: Una nueva forma de enfocar las sesiones prácticas Blending learning: A new way of approaching practical sessions
}

\author{
Cristina Casto-Rebollo ${ }^{\ddagger 1}$, Eugenio Martinez-Paredes ${ }^{\ddagger 1}$, Silvia Martínez-Llorens ${ }^{1}$, José S.Vicente ${ }^{1}$, Francisco Marco- \\ Jiménez ${ }^{1}$, David S. Peñaranda ${ }^{1 *}$ \\ cricasre@posgrado.upv.es; eumarpa@upvnet.upv.es; silmarll@dca.upv.es; jvicent@upv.es; fmarco@upv.es; dasncpea@upv.es, \\ † Los autores contribuyeron de igual manera \\ ${ }^{1}$ Departamento de Ciencia Animal \\ Universitat Politècnica de València \\ Valencia, España
}

Resumen-Las competencias son elementos muy apreciados por los empleadores, en especial las relacionadas con el pensamiento práctico. Esta realidad es más evidente en las sesiones prácticas de laboratorio, donde los alumnos deben aplicar conocimientos teóricos a casos prácticos. En el actual trabajo se pretende implantar la metodología docente "Blended learning" que combina docencia presencial con on line, con el fin de favorecer la adquisición de la competencia transversal "Aplicación y pensamiento práctico", así como una mayor interiorización de los conceptos aprendidos. La metodología docente se implantó en alumnos de grado, en concreto en las sesiones de laboratorio de la asignatura: Fisiología Animal y Humana (11109) del segundo curso del Grado en Biotecnología de la Universitat Politècnica de València. Los resultados obtenidos mostraron que la implantación de la metodología docente permitió desarrollar el pensamiento práctico, ya que la nota media categórica fue de "A: Excelente". Además, se observó una mejora en la calificación de la nota del examen teórico-práctico, lo que indica una mejor interiorización de los conceptos impartidos. Por tanto, se puede concluir que la metodología "Blended learning" ha sido una metodología eficaz para no sólo mejorar la adquisición de la competencia transversal, sino también para la calificación académica.

Palabras clave: Docencia Semi-presencial, pensamiento práctico, prácticas de laboratorio

Abstract- Competencies are highly appreciated by employers, especially those related to practical thinking. This reality is more evident in laboratory practical sessions, where students should apply theoretical knowledge to practical cases. The current work aims to apply the teaching methodology "Blended learning" that combines face-to-face teaching with online, in order to improve the acquisition of transversal competence "Application and practical thinking", as well as a greater internalization of learned concepts. The teaching methodology was implemented in undergraduate students, specifically in the laboratory sessions of the subject: Animal and Human Physiology (11109) of the second year of the Degree in Biotechnology at the Universitat Politècnica de València. The results obtained showed that the application of the teaching methodology allowed the development of practical thinking, since the categorical average grade was "A: Excellent". In addition, there was an improvement in the qualification of the note of the theoretical-practical evaluation, indicating a better internalization of the learned concepts. Therefore, it can be concluded that the "Blended learning" methodology has been an effective methodology for not only improving the acquisition of transversal competence, but also for academic qualification.

Keywords: Blending learing, practical thinking, laboratory seasons

\section{INTRODUCCIÓN}

Cada vez es mayor la tendencia de un aprendizaje centrado en el estudiante, lo que supone un cambio en el papel del maestro. El docente ya no se dedica únicamente a transmitir conocimientos o a instruir, sino también a orientar el proceso educativo en ambientes en los cuales el estudiante sea capaz de identificar y decidir lo que quiere aprender y las condiciones en que va a hacerlo (Roger \& Ruiz, 2014).

En este sentido, la creación de ambientes docentes que promuevan el pensamiento práctico es una herramienta de gran valor, no sólo porque le obliga a ser estratégicos y dirigir su motivación, sino también para el desarrollo de habilidades, destrezas e incluso actitudes que les permitan la adquisición de esta competencia (de la Cruz Flores \& Hernández, 2014); Mosconi et al., 2019). Un aprendizaje donde el alumno debe encontrar soluciones argumentadas acomodándose a los recursos disponibles, lo convierte en un sujeto activo, que es capaz de establecer metas y monitorizar sus avances (del Valle López \& Fernández, 2008), favoreciendo por tanto su autonomía (Núñez et al., 2006).

Esta mejora de la autonomía, se basa en que el alumno es capaz de autorregular su aprendizaje, sustentándose en los siguientes procesos metacognitivos (Gauthier, 2014):

- Permite auto valorar las tareas realizadas, al establecer metas en un tiempo limitado.

- Permite evaluar sus propios conocimientos y habilidades, sacando a la luz sus fortalezas y debilidades.

- Ayuda a planificarse en una situación real

- Permite monitorear el desarrollo de la actividad

- Reflexionar sobre el grado en que su enfoque funciona, con el fin de realizar ajustes

El objetivo es que los alumnos transiten de aprendices a expertos, y para ello es necesario la creación de un andamiaje que permita al alumno interiorizar los conceptos adquiridos y alcanzar un aprendizaje más profundo (Joerns, 2011). Para la creación de este andamiaje, es necesario la previa formación del alumno, siendo preferible que ésta sea on line, sin las restricciones de la clase presencial. Con este fin, las TIC son una herramienta de gran valor. Actualmente, las TIC se están convirtiendo en una de las variables esenciales de los escenarios 
formativos (Gibelli et al., 2012) y, en concordancia, los investigadores de la educación están centrando sus estudios en una enseñanza y, y aprendizaje mediadas por TIC.

La enseñanza a distancia mediada por TIC favorece el seguimiento de metas personales, la libre búsqueda de información y resolución de diferentes situaciones problemáticas, de acuerdo con las diferencias individuales de los estudiantes (Jacobson \& Archodidou, 2000). Por tanto, en el actual trabajo se propone una docencia conocida como Blended learning o enseñanza semi-presencial, siendo una metodología docente de gran adaptabilidad que permite crear el ambiente adecuado para un aprendizaje más autónomo que fomente una mejor interiorización de los conceptos aprendidos, y, por tanto, un mayor éxito a la hora de aplicar los conocimientos teóricos a casos prácticos.

La incorporación en el aula de la metodología docente Blended learning ha ido adquiriendo importancia paralelamente a la irrupción de las TIC en la enseñanaza superior. Esta metodología docente combina una enseñanaza on line con una presencial, pero no está definido qué nivel de docencia on line debe incluir. De cualquier manera, no son relevantes los porcentajes de los dos tipos de docencia, sino que se realice una remodelación real a todos los niveles (recursos, objetivos de aprendizaje, materiales..) de la actividad propuesta para alcanzar una efectiva integración de la docencia on line y presencial (Garrison \& Kanuka, 2004). Este tipo de docencia no debe confundirse con la docencia inversa, donde el alumno tiene actividades tanto pre como post clase presencial, y durante la clase presencial se discute o se profundiza sobre los materiales trabajados. La metodología docente Blended learning no incluye una evaluación post clase presencial, sino que es durante la misma clase presencial, en función de si los alumnos son capaces de resolver los problemas a los que tienen que enfrentarse.

Por último, la versatilidad entre la parte on line y presencial de este tipo de docencia, permite adaptarse a diferentes ambientes académicos. Característica de gran utilidad en situaciones como la actual, que a causa del COIVD-19 la metodología docente ha tenido que modificarse para adaptarse a las restricciones de presencialidad (Area-Moreira et al., 2021).

\section{CONTEXTO}

Una de las exigencias que plantea la sociedad a la universidad es potenciar y facilitar la inserción socio-laboral de los futuros egresados. Para ello, el desarrollo de las competencias es un elemento que cada día es más apreciado por los empleadores, siendo de especial relevancia las relacionadas con el pensamiento crítico o práctico (Yaoxiang, 2019). La implantación de las competencias en el mundo educativo, no siempre ha supuesto un cambio en la metodología docente, imposibilitando en muchas ocasiones alcanzar los objetivos inicialmente planteados. Esta realidad es más evidente en el campo de las ciencias, especialmente, en docencia práctica de laboratorio (Cano, 2019). Investigaciones en este campo apuntan a una falta de claridad en los objetivos y la poca capacidad para relacionar teoría y práctica (Barberà, 1996; Hodson, 1994). Las sesiones prácticas suelen ser actividades cerradas y simplemente plantean una serie de pasos (Velandia et al., 2011), y cada vez son más los estudios que resaltan la importancia del ambiente de aprendizaje y la metacognición en actividades de laboratorio (Cooper et al., 2008; Llorens Molina et al., 2012).
En el actual trabajo se pretenden crear ambientes de trabajo y actividades que propicien la adquisición de la competencia transversal "Aplicación y pensamiento práctico", que consiste en aplicar los conocimientos teóricos y establecer el proceso a seguir para alcanzar determinados objetivos, llevar a cabo experimentos y analizar e interpretar datos para extraer conclusiones (Universitat Politècnica de València, Competencias transversales, Aplicación y Pensamiento práctico). Para ello se plantea implantar la metodología docente: Blended learning o enseñanza semi-presencial.

La metodología docente propuesta se aplicará en alumnos de grado, en concreto en las sesiones de laboratorio asignatura: Fisiología Animal y Humana (11109) del segundo curso del Grado en Biotecnología de la Universitat Politècnica de València (UPV) (106 alumnos). Los alumnos durante la realización de las actividades se limitan a seguir el guion de la actividad o preguntar constantemente al profesor, sin un interés real en finalizar correctamente la actividad o comprenderla, ya que la calificación de la actividad suele depender de una evaluación teórica-práctica posterior. Por tanto, por norma general, los alumnos no alcanzan los resultados de aprendizaje y competencias propuestas en la actividad.

Con el fin de cambiar esta actitud, se plantea un cambio de metodología docente donde los alumnos tengan que realizar previo a la clase presencial y de carácter obligatorio, una serie de actividades de forma on line. Una vez en la sesión presencial, los alumnos serán evaluados tanto del desarrollo de la competencia transversal "Aplicación y pensamiento práctico", como los conocimientos teórico-prácticos de la actividad.

Por tanto, en base a esta metodología docente se plantean las siguientes hipótesis:

- La preparación de los contenidos de forma on line previo a la sesión presencial permite el desarrollo y la aplicación del pensamiento práctico.

- La necesidad de aplicar los conocimientos teóricos a un caso práctico refuerza al alumno ayuda a la adquisición de un aprendizaje más profundo.

\section{A. Objetivo General}

El estudiante, en la vida real, necesita estar preparado para hacer frente a situaciones en las que no basta aplicar recetas o fórmulas. Es por ello, que en el actual trabajo de investigación se propone un cambio de metodología para crear un ambiente docente que desarrolle la competencia "Aplicación y pensamiento práctico". En este sentido, las acciones planteadas están dirigidas a que los alumnos sepan adaptarse a nuevas situaciones, tomar decisiones y, consecuentemente, ACTUAR. Con este fin, se ha diseñado la incorporación de la metodología Blending learning en las sesiones de laboratorio de la asignatura Fisiología Animal y Humana con los siguientes objetivos específicos:

- Diseño de una propuesta docente que promueva la aplicación de los conocimientos teóricos a casos prácticos.

- Proponer actividades que estimulen un aprendizaje más profundo, favoreciendo que este aprendizaje sea a más largo plazo.

- Diseñar una rúbrica de evaluación que permita al docente calificar el desarrollo de la competencia transversal.

\section{DESCRIPCIÓN}

La asignatura seleccionada para implantar el proyecto docente es la siguiente: 
-Fisiología Animal y Humana (11109) del Grado en Biotecnología de la UPV.

Fisiología Animal y Humana es una asignatura de $2^{\circ}$ curso y está considerada de formación básica. Consta de un total de 9 créditos, 6 de teoría y 3 de prácticas. La medía de alumnos matriculados de los últimos 5 años es de unos 100 estudiantes, que se dividen en tres grupos de teoría: A y B en castellano, y un grupo de "Alto Rendimiento Académico (ARA)" que usa como lengua vehicular el inglés. A su vez los tres grupos de teoria, se subdividen en 5 grupos de prácticas y seminarios (A12, B1-2, ARA).

Las variables que será necesario medir/controlar la competencia transversal:

- Evolución de la puntuación de la rúbrica a lo largo de las sesiones prácticas.

- Evolución de la valoración de la competencia a lo largo de las sesiones prácticas.

Mientras que para valorar el aprendizaje profundo se utilizó:

- Correlación entre el nivel de competencia alcanzado y la nota en la práctica y/o teórica.

El instrumento/s de recogida de información

- Rúbrica

- Actividades y exámenes

Las actividades on line se llevaban a cabo previo a la clase presencial, estando disponibles una semana antes y siendo el tiempo límite de entrega 48 horas previo a la actividad presencial. La rúbrica se repartía en papel de forma individual al inicio de la clase presencial, y el profesor al finalizar la misma la rellenaba y procedía a su recogida. También al finalizar las actividades de la clase presencial, se llevaba a cabo la evaluación académica de cada sesión.

\section{B. Descripción de la Metodología}

Con el fin de que el alumno sea capaz de desarrollar la actividad presencial de forma autónoma, es necesario que el alumno se forme previamente del contenido teórico de la actividad y de los pasos a realizar durante la misma. Por tanto, se desarrollaron actividades on line, previo a la clase presencial, que permitan la adquisición de los contenidos necesario para realizar la actividad.

Para la actividad on line se divide en las siguientes secciones (Figura 1):

- Presentación de la actividad: Con la descripción breve de los contenidos a trabajar, los diferentes resultados de aprendizaje y competencias transversales planteadas, y finalmente una figura o tabla con las secuencias de aprendizaje.

- Introducción Teórica-Práctica: Contenido teórico relacionado con la actividad con enlaces a documentos, vídeos o páginas web que permitan a los alumnos realizar un aprendizaje más profundo del contenido teórico de la actividad. Además, este apartado incluye un video realizado por el equipo de investigación docente donde se describe con detalle los pasos a realizar durante la actividad. Con el fin de que los alumnos pueden tener una total autonomía durante la realización de la actividad, también incluirá un guion escrito de los pasos a realizar en la actividad.

- Actividad: El alumno al finalizar el módulo debe realizar una actividad (opción múltiple, imagen interactiva, pregunta calculada, verdadero-falso..), que no computará para la nota final, pero si será de obligatorio cumplimiento. Se podrá repetir todas las veces que sea necesario, con el fin de que los alumnos afiancen los conceptos trabajados.

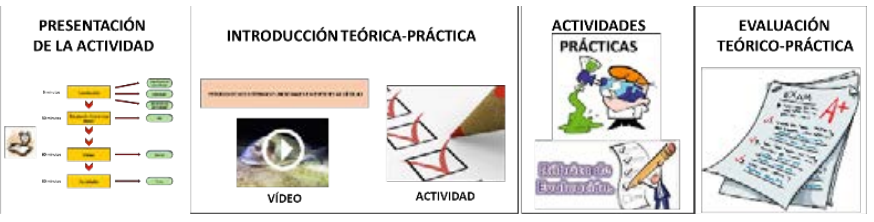

Figura 1. Secuencia de las tareas a desarrollar

Una vez en la clase presencial, los alumnos deberán realizar las actividades propuestas de forma autónoma. Con el fin de evaluar su capacidad de aplicar el pensamiento práctico, se utilizará la siguiente rúbrica, siendo evaluados los alumnos a medida que van realizando la actividad.

Tabla 1. Rúbrica para la evaluación del proceso de aprendizaje

\begin{tabular}{|c|c|c|c|c|c|}
\hline Categoria & 1 & 2 & 3 & 4 & Nota \\
\hline $\begin{array}{c}\text { Preparación de } \\
\text { la práctica }\end{array}$ & $\begin{array}{c}\text { El alumno no tiene } \\
\text { preparada la } \\
\text { práctica en } \\
\text { absoluto }\end{array}$ & $\begin{array}{l}\text { El alumno ha } \\
\text { trabajado los material } \\
\text { antes de la clase } \\
\text { presencial de forma } \\
\text { on-line, pero no tiene } \\
\text { el material necesario } \\
\text { para su realización } \\
\text { (bata, manuales, } \\
\text { actividades on line.) }\end{array}$ & $\begin{array}{l}\text { El alumno ha trabajado } \\
\text { los material antes de la } \\
\text { clase presencial de } \\
\text { forma on-line, los } \\
\text { comprende, pero no es } \\
\text { capaz de aplicar los } \\
\text { contenidos teóricos de } \\
\text { forma práctica }\end{array}$ & $\begin{array}{c}\text { El alumno comprende } \\
\text { perfectamente los } \\
\text { contenidos impartidos } \\
\text { previo a la clase } \\
\text { presencial, los sabe aplicar } \\
\text { de forma práctica y tiene } \\
\text { todo el material necesario } \\
\text { para su realización }\end{array}$ & \\
\hline $\begin{array}{c}\text { Nivel de } \\
\text { intervención } \\
\text { del } \\
\text { profesorado }\end{array}$ & $\begin{array}{l}\text { El alumno es } \\
\text { incapaz de } \\
\text { proseguir la } \\
\text { práctica sin la } \\
\text { intervención del } \\
\text { profesorado }\end{array}$ & $\begin{array}{l}\text { El alumno necesita el } \\
\text { asesoramiento del } \\
\text { profesorado en varias } \\
\text { ocasiones pero } \\
\text { presenta cierta } \\
\text { autonomia }\end{array}$ & $\begin{array}{l}\text { El alumno necesita } \\
\text { alguna ayuda puntual, } \\
\text { pero es capaz de } \\
\text { realizar la práctica por } \\
\text { si solo }\end{array}$ & $\begin{array}{l}\text { El alumno es totalmente } \\
\text { autónomo, y realiza la } \\
\text { práctica por si sololo }\end{array}$ & \\
\hline $\begin{array}{c}\text { Finalización de } \\
\text { la práctica con } \\
\text { éxito }\end{array}$ & $\begin{array}{l}\text { El alumno soblo es } \\
\text { capaz de finalizar el } \\
25 \% \text { o menos de la } \\
\text { prática con exito }\end{array}$ & $\begin{array}{l}\text { El alumno es capaz de } \\
\text { finalizar hasta } 50 \% \text { de } \\
\text { la práctica con éxito }\end{array}$ & $\begin{array}{c}\text { El alumno finaliza con } \\
\text { exito hasta el } 75 \% \text { de } \\
\text { la práctica }\end{array}$ & $\begin{array}{l}\text { El alumno es capaz de } \\
\text { finalizar el } 100 \% \text { de la } \\
\text { práctica con exito }\end{array}$ & \\
\hline Nota final & & & & & \\
\hline
\end{tabular}

Para cada una de las categorías (Preparación de la práctica, nivel de intervención del profesorado, finalización del a práctica) se estableció una puntuación numérica del 1 al 4, siendo 1 el mínimo y 4 la máxima puntuación. La puntuación total obtenida en la rúbrica se utilizará para la valoración de la competencia transversal; siendo; A: Excelente (11-12), B: Adecuado (8-10), C: En desarrollo (5-7), y D: No alcanzado (14).

Una vez finalizada la actividad, los alumnos se someten a una prueba teórico-práctica, que consta de preguntas tipo test o de respuesta abierta, relacionado con las actividades que acaban de realizar durante la clase presencial o de forma on line desde casa.

- Prácticas: Las sesiones de prácticas estaban dividas en tres grupos; G1: Modelos animales, G2: Histología y G3: Reproducción. En el grupo G1 se evaluaban por separado la teoría de dos modelos animales diferentes: El modelo conejo y el modelo dorada. Al igual que en el grupo G3, donde se evaluaban por un lado la fisiología reproductiva del macho (Espermiograma), y por el otro la fisiología reproductiva de la hembra (Recuperación ovocitaria). Para ambos grupos (G1 y G2) la nota de la práctica es la media del examen teórico práctico de cada una de las partes. En el grupo G2 se realizó un único examen teórico práctico con los conceptos de Histología.

- Análisis estadísticos: Las diferencias entre las notas de cada una de las prácticas con la calificación de la competencia transversal se evaluaron mediante la prueba no paramétrica de Mann-Whitney U. Las diferencias entre el porcentaje de alumnos con una determinada calificación de la competencia transversal a lo largo de cada una de las prácticas se evaluaron usando el estadístico F de Fisher. Las diferencias observadas entre las variables analizadas se consideraron relevantes cuando el p-valor de estas diferencias era inferior a 0.05. Las correlaciones se calcularon usando el coeficiente de correlación de Spearman. 


\section{Resultados}

La valoración de la competencia transversal se calcula mediante la evaluación de tres capacidades de los alumnos: a) Preparación de la Práctica, b) Autonomía durante la práctica, y c) Grado de ejecución de la práctica. En todas las prácticas, la mayoría de los alumnos han obtenido la máxima puntuación en el apartado de preparación (Figura 2.a). Esto nos indica que todos los alumnos han trabajado los conceptos teóricos antes a la práctica, y que todos han llevado los materiales necesarios para su desarrollo. Diferencias relevantes ( $p$-valor $<4 \mathrm{e}-15)$ se observaron en el apartado que evaluaba la autonomía, ya que el número de alumnos que presentaban la máxima puntuación disminuía a medida que avanzaban las prácticas (Figura 2.b). Las prácticas más complicadas se distribuyeron al final, con lo que posiblemente esta sea la razón de la bajada en la calificación de esta capacidad. De hecho, la calificación obtenida en el grado de ejecución de la práctica (Figura 2.c), muestra una tendencia similar con diferencias significativas ( $\mathrm{p}$ valor $<2 \mathrm{e}-5$ ) entre la práctica G1 y G2-3.

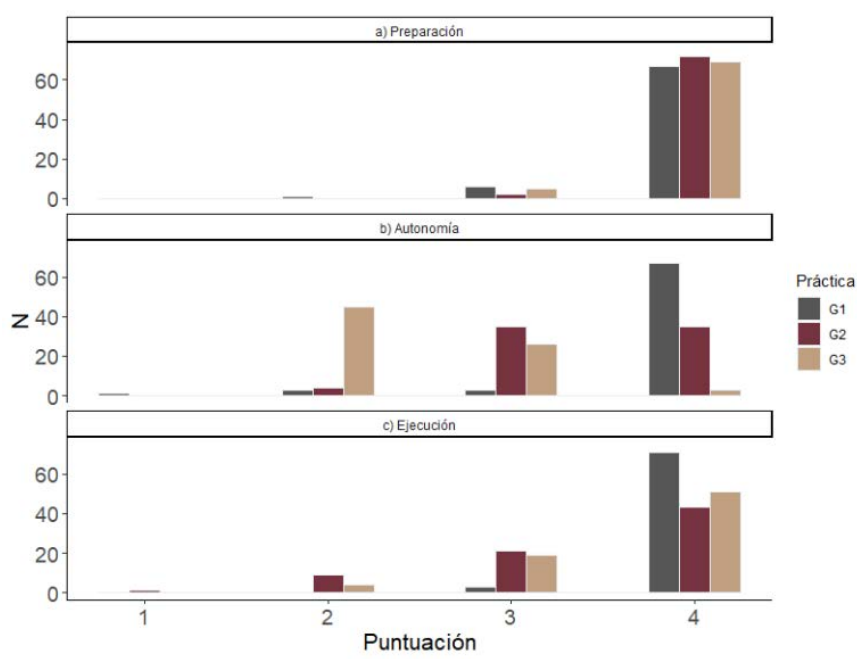

Figura 1. Porcentaje de estudiantes que han obtenido una puntuación de 1 a 4 en cada categoría de la rúbrica. G1, G2, G3 hacen referencia a cada uno de los tres grupos de sesiones de práctica realizados. a) Preparación. Categoría que marca la preparación del alumno previa a la clase, así como la disponibilidad del material necesario en la práctica. b) Autonomía. Categoría que indica el grado de autonomía del alumnado, evaluado según el número de intervenciones del profesor. c) Ejecución. Categoría que determina el éxito de la práctica, evaluado de $0 \%$ (no realizada) a 100\% (finalizada completamente).

Cuando se evaluó el porcentaje de alumnos con una determinada calificación para la competencia transversal a lo largo de las prácticas, la gran mayoría independientemente de la práctica, se encontraban en la calificación de A y B (Tabla 2). Los porcentajes de cada categoría, aumentando los alumnos en la categoría B y disminuyendo los de la categoría A, vuelven a confirmar la mayor dificultad de las prácticas distribuidas al final de la asignatura.

Tabla 2. Porcentaje de alumnos con una determinada valoración de la competencia transversal $(\mathrm{A}, \mathrm{B}, \mathrm{C}, \mathrm{D})$ en las diferentes prácticas $(\mathrm{G} 1, \mathrm{G} 2, \mathrm{G} 3)$

\begin{tabular}{|c|c|c|c|c|}
\hline \multirow{5}{*}{ 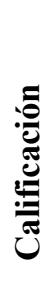 } & & G1 & $\mathrm{G} 2$ & G3 \\
\hline & A: Excelente & 87.74 & 70.75 & 32.43 \\
\hline & B: Adecuado & 11.32 & 29.25 & 67.57 \\
\hline & C: En desarrollo & 0.94 & 0 & 0 \\
\hline & D: No alcanzado & 0 & 0 & 0 \\
\hline
\end{tabular}

Sin embargo, cuando se observó la nota obtenida en función de la valoración obtenida en la competencia transversal, se observó una tendencia alcista tanto en los alumnos con la competencia A como B (Figura 3). Esto nos indica que los alumnos iban mejorando el proceso de aprendizaje a medida que se adaptaban a la nueva metodología docente, incluso cuando la dificultad de las prácticas era superior. Además, existió una relación entre las calificaciones del proceso de aprendizaje y las notas del examen para la práctica G1 ( $p$-valor $=0.02$ ). Alumnos calificados como "A: Excelente" para el pensamiento práctico fueron los qué obtuvieron las notas más altas en el examen teórico posterior a la práctica. Esto sugiere que la metodología propuesta ayuda no sólo a desarrollar el pensamiento práctico sino además interiorizar los conceptos. No obstante, esta relación no se observó en las otras prácticas, donde las notas del examen teórico son similares entre los alumnos calificados como A y B. Posiblemente, este hecho se debe a que el grado de dificultad de las prácticas era mayor, y los alumnos necesitaban un mayor asesoramiento del profesorado para la consecución de la práctica (Figura 2c). Una mayor intervención del profesorado supuso una bajada en el porcentaje de alumnos calificados como A: Excelente, y un aumento de los calificados como B: Adecuado (p-valor $<2 \mathrm{e}-14)$. No obstante, como se observa en la figura 3 , el proceso de aprendizaje de la materia fue mejorando a medida que avanzaban las prácticas, como demuestra las notas medias de los exámenes teóricos de los alumnos con valoración A y B que mejoraron a medida a medida que avanzaban las prácticas (Figura 3; p-valor de la diferencia de medias entre G1 y G3 de 0.007).

En base a los resultados, podemos concluir que es cierta la hipótesis que la metodología docente estimuló el desarrollo del pensamiento práctico, ya que la nota media categórica teniendo en cuenta todas las prácticas y todos los alumnos es de "A: Excelente". Esta media se calculó usando la puntuación total de la rúbrica de las tres prácticas. Para ello se hizo el sumatorio total y se determinó a que categoría correspondía esa valoración numérica total; A: Excelente, B: Adecuado, C: En desarrollo y D: No alcanzado.

\section{Práctica}




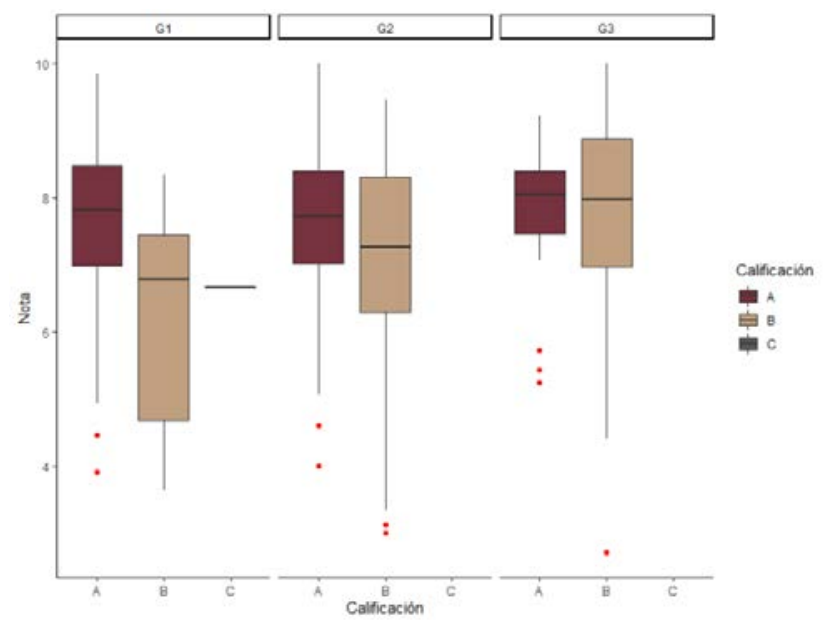

Figura 3. Nota obtenida en la práctica en función de la valoración de la competencia transversal "Pensamiento práctico". G1, G2, G3 hacen referencia a cada uno de los tres grupos de sesiones de práctica realizados. A, B, C son las calificaciones del proceso de aprendizaje. La nota de la práctica va de 0 a 10. Los puntos rojos son alumnos anómalos para la distribución a la que pertenecen.

Por otro lado, el aumento de la calificación de la nota de la evaluación teórico-práctica tanto en los alumnos valorados en la categoría A como la $\mathrm{B}$, nos lleva a pensar que también es cierta la hipótesis que la necesidad de aplicar los conocimientos teóricos a un caso práctico refuerza al alumno a interiorizar los conceptos aprendidos.

Los objetivos planteados se han cumplido, ya que la media categórica de la competencia transversal fue de "A: Excelente", por lo tanto, se puede afirmar que la metodología docente aplicada "Blending learning" ha sido efectiva a la hora aplicar los conocimientos teóricos a un caso práctico. Por otro lado, las actividades diseñadas han estimulado un aprendizaje más profundo, favoreciendo una mejor interiorización de los conceptos, como se puede comprobar al observar que los alumnos con una calificación de $\mathrm{A}$ en la competencia transversal obtuvieron notas superiores a los alumnos con una calificación de B. Finalmente, la rúbrica diseñada ha resultado ser efectiva para medir la competencia transversal, aunque una calificación de 10 para cada habilidad en lugar de 1 a 4, hubiera facilitado una mejora gradación del nivel de desarrollo de la competencia, y por tanto mejor valoración de la competencia transversal: "Aplicación y pensamiento práctico".

\section{CONCLUSIONES}

Se puede concluir que la implantación de la metodología docente ha permitido desarrollar la competencia transversal: "Aplicación y pensamiento práctico", lo que ha supuesto una mejora en el aprendizaje de los conceptos impartidos, mejorando la nota obtenida en la evaluación teórico-práctica. Por tanto, la metodología docente "Blending learning" se ha demostrado como un método eficaz, que además presenta la ventaja de una gran flexibilidad a situaciones y necesidades docentes muy diversas al combinar docencia presencial con una on line.

\section{Agradecimientos}

Agradecer a los profesores por su esfuerzo en implantar esta nueva metodología docente. También nos gustaría agradecer a agradecer el apoyo del Vicerrectorado de Estudios, Calidad y Acreditación de la Universitat Politècnica de València al proyecto PIME/20-21/213 "Estrategias para favorecer la autorregulación en el aprendizaje de competencias" y al proyecto PIME/18-19/74 “Aprendizaje basado en proyectos para fomentar la visión profesional en el máster de Acuicultura".

\section{REFERENCIAS}

Area-Moreira, M., Bethencourt-Aguilar, A., Martín-Gómez, S., \& San Nicolás-Santos, M. B. (2021). Análisis de las políticas de enseñanza universitaria en España en tiempos de Covid-19. La presencialidad adaptada. Revista de Educación a Distancia (RED), 21(65).

Barberà O., V. P. (1996). El trabajo práctico en la enseñanza de las ciencias: una revisión. Enseñanza de Las Ciencias: Revista de Investigación y Experiencias Didácticas, 14(3), 365-379. https://www.raco.cat/index.php/Ensenanza/article/downl oad/21466/93439

Cano, M. F. C. (2019). ABP: repensando los laboratorios de química. REDU: Revista de Docencia Universitaria, 17(2), 4.

Carlos Núñez, J., Solano, P., González-Pienda, J. A., \& Rosário, P. (2006). EL APRENDIZAJE AUTORREGULADO COMO MEDIO Y META DE LA EDUCACIÓN. http://www.cop.es/papeles

Cooper, M. M., Sandi-Urena, S., \& Stevens, R. (2008). Reliable multi method assessment of metacognition use in chemistry problem solving. Chemistry Education Research and Practice, 9(1), 18-24.

Gabriela de la Cruz Flores y Luis Felipe Abreu Hernández. (2014). Rúbricas y autorregulación: pautas para promover una cultura de la autonomía en la formación profesional terciaria. Revista de Docencia Univesitaria, 12(1), 31-48. https://doi.org/10.1163/_3_SIM_00374

Garrison, D. R., \& Kanuka, H. (2004). Blended learning: Uncovering its transformative potential in higher education. https://doi.org/10.1016/j.iheduc.2004.02.001

Gauthier, L. M. (2014). Book Review How Learning Works: 7 Research-Based Principles for Smart Teaching. Journal of the Scholarship of Teaching and Learning, 14(1), 126129. https://doi.org/10.14434/josotl.v14i1.4219

Gibelli, T., e, A. C.-I. J. N. de T., \& 2012, undefined. (n.d.). Estrategias de aprendizaje y autorregulación usando TIC. In sedici.unlp.edu.ar. Retrieved July 3, 2020, from http://sedici.unlp.edu.ar/handle/10915/26521

Hodson, D. (1994). Hacia un enfoque más crítico del trabajo de laboratorio. Enseñanza de Las Ciencias: Revista de Investigación y Experiencias Didácticas, 12(3), 299-313. https://scholar.google.es/scholar?hl=ca\&as_sdt $=0 \% 2 \mathrm{C} 5$ $\& \mathrm{q}=$ Hacia + un + enfoque + más + crítico + del + trabajo + de + la boratorio\&btnG $=$

Jacobson, M. J., \& Archodidou, A. (2000). The Design of Hypermedia Tools for Learning: Fostering Conceptual Change and Transfer of Complex Scientific Knowledge. In THE JOURNAL OF THE LEARNING SCIENCES 
(Vol.

9

Issue

2).

https://www.tandfonline.com/doi/abs/10.1207/s1532780 9j1s0902_2

Joerns, J. L. (2011). Aprendizaje como reconfiguración de agencia. Revista de Estudios Sociales, 40, 33-43. https://doi.org/10.7440/res40.2011.04

Llorens Molina, J. A., Llorens De Jaime, J. M., \& Sanz Berzosa, I. (2012). La caracterización del ambiente de aprendizaje en un laboratorio de química general mediante métodos de investigación social. Enseñanza de Las Ciencias. Revista de Investigación y Experiencias Didácticas, 30(1), 5-22.

del Valle López, Á., \& Fernández, N. V. (2008). Aprendizaje basado en problemas: una propuesta metodológica con futuro. In El aprendizaje basado en problemas (ABP): una propuesta metodológica en Educación Superior (pp. 2734).

Narcea. https://ialnet.unirioja.es/servlet/articulo? codigo $=27490$ 34

Mosconi, E. B., Bustichi, G. S., Pollicina, L. M., \& Varela, J. N. (2019). Estrategias de autorregulación académica. Revista de La Facultad de Odontología.
Roger, J., \& Ruiz, R. (2014). Los recursos tic favorecedores de estrategias de aprendizaje autónomo: el estudiante * autónomo y autorregulado the tic resources favoring independent learning strategies: student self-regulated and self-Ensayos. In: 19 agosto (Vol. 5, Issue 2). http://revistas.uladech.edu.pe/index.php/increscendo/arti cle/view/403

Universitat Politècnica de Valencia, Competencias transversales, Aplicación y Pensamiento Práctico. http://www.upv.es/contenidos/COMPTRAN/info/95471 5normalc.html.

Velandia, M. A. A., Morales, F. H. F., \& Duarte, J. E. (2011). Utilización de material didáctico para la enseñanza de los conceptos de ciencia y tecnología en niños. Revista de Investigación, Desarrollo e Innovación, 2(1), 35-43.

Yaoxiang, L. (2019). Practical Thinking and Innovative Strategy of Diagnosing and Improving Teaching Work in Higher Vocational Colleges. Advances in Educational Technology and Psychology, 3(1), 80-88. 